\title{
Lupus and proliferative nephritis are PAD4 independent in murine models
}

\author{
Rachael A. Gordon, ${ }^{1}$ Jan M. Herter, ${ }^{2}$ Florencia Rosetti, ${ }^{2}$ Allison M. Campbell, ${ }^{3}$ Hiroshi Nishi, ${ }^{2}$ \\ Michael Kashgarian, ${ }^{4}$ Sheldon I. Bastacky, ${ }^{5}$ Anthony Marinov, ${ }^{1}$ Kevin M. Nickerson, ${ }^{1}$ \\ Tanya N. Mayadas, ${ }^{2}$ and Mark J. Shlomchik ${ }^{1,3,6}$ \\ 'Department of Immunology, University of Pittsburgh School of Medicine, Pittsburgh, Pennsylvania, USA. ${ }^{2}$ Department \\ of Pathology, Brigham and Women's Hospital, and Harvard Medical School, Boston, Massachusetts, USA. ${ }^{3}$ Department \\ of Immunobiology, ${ }^{4}$ Department of Pathology, Yale University School of Medicine, New Haven, Connecticut, USA. \\ ${ }^{5}$ Department of Pathology, University of Pittsburgh School of Medicine, Pittsburgh, Pennsylvania, USA. ${ }^{6}$ Department of \\ Laboratory Medicine, Yale University School of Medicine, New Haven, Connecticut, USA.
}

Though recent reports suggest that neutrophil extracellular traps (NETs) are a source of antigenic nucleic acids in systemic lupus erythematosus (SLE), we recently showed that inhibition of NETs by targeting the NADPH oxidase complex via cytochrome b-245, $\beta$ polypeptide (cybb) deletion exacerbated disease in the MRL.Fas ${ }^{\text {Ip }}$ lupus mouse model. While these data challenge the paradigm that NETs promote lupus, it is conceivable that global regulatory properties of cybb and cybbindependent NETs confound these findings. Furthermore, recent reports indicate that inhibitors of peptidyl arginine deiminase, type IV (Padi4), a distal mediator of NET formation, improve lupus in murine models. Here, to clarify the contribution of NETs to SLE, we employed a genetic approach to delete Padi4 in the MRL.Fas ${ }^{\mid p r}$ model and used a pharmacological approach to inhibit PADs in both the anti-glomerular basement membrane model of proliferative nephritis and a human-serum-transfer model of SLE. In contrast to prior inhibitor studies, we found that deletion of Padi4 did not ameliorate any aspect of nephritis, loss of tolerance, or immune activation. Pharmacological inhibition of PAD activity had no effect on end-organ damage in inducible models of glomerulonephritis. These data provide a direct challenge to the concept that NETs promote autoimmunity and target organ injury in SLE.

Authorship note: R.A. Gordon, J.M. Herter, and F. Rosetti contributed equally to this work. M. Kashgarian and S.I. Bastacky contributed equally to this work. T.N. Mayadas and M.J. Shlomchik contributed equally to this work.

Conflict of interest: TNM is on the advisory board of Biothera and received research support from the company for work unrelated to the present study.

Submitted: January 18, 2017

Accepted: April 10, 2017

Published: May 18, 2017

\section{Reference information:}

JCI Insight. 2017;2(10):e92926.

https://doi.org/10.1172/jci.

insight. 92926

\section{Introduction}

Systemic lupus erythematosus (SLE) is a multisystem autoimmune disease characterized by loss of tolerance to nuclear antigens, resulting in the formation of autoantibodies against DNA, RNA, and ribonuclear proteins, rampant immune activation, and tissue destruction (1). Though the sources of autoantigens in SLE are not known, the release of cellular contents from living or dying cells is considered the most likely possibility (2).

Neutrophil extracellular traps (NETs) are extruded DNA structures coated with neutrophil granule proteins (3). Early reports suggest that decondensed nuclear DNA is the major constituent of NETs and that the neutrophil dies at the conclusion of this process (4). More recently, nuclear DNA externalization without concomitant cell lysis (5) and extrusion of mitochondrial DNA (6-8) have been described. Classical NET formation in humans and mice is dependent on NADPH oxidase-generated reactive oxygen species (ROS) $(4,9)$, though rapid NADPH oxidase-independent NET formation has been reported $(5$, 10). Peptidyl arginine deiminase, type IV (Padi4), an enzyme that citrullinates histones and facilitates chromatin decondensation, is critical for NET formation (11-16).

Several lines of evidence suggest that NETs may be a primary and nonredundant source of self-antigen in SLE. NET-like structures are found in the skin and kidneys of SLE patients and SLE-prone mice (17-20), while NET degradation is impaired in a minor subset of individuals with lupus $(21,22)$. Abnormal low-density granulocyte (LDG) populations identified in peripheral blood mononuclear cell (PBMC) fractions isolated from SLE cohorts have an increased propensity to form NETs in vitro, potentially enhancing exposure to autoantigens and immunostimulatory molecules $(17,23)$. Neutrophils from SLE patients can activate plasmacytoid dendritic cells (pDCs) to produce type I interferon (IFN) upon culture in vitro, a phenomenon attributed specifically to NET formation $(18,24)$. Concordantly, anti-ribonuclear protein (anti-RNP) antibodies, which 
are present in a subgroup of SLE patients, can induce in vitro NETs from SLE but not normal neutrophils, in a process dependent on Fc $\gamma$ RIIA, ROS, and TLR7 (18). Activating Fc $\gamma$ receptors (Fc $\gamma$ Rs) are critical for the pathogenesis of SLE nephritis (25), and neutrophil Fc $\gamma$ Rs promote renal injury (26), leading to the possibility that Fc $\gamma$ R-mediated NET formation contributes to end-organ injury. More recently, 2 groups reported that antiRNP antibodies and immune complexes (ICs) can induce the externalization of immunostimulatory oxidized mitochondrial DNA $(7,8)$. While SLE LDGs release oxidized mitochondrial DNA (8) and anti-oxidized mitochondrial DNA autoantibodies are elevated in pediatric SLE patients (7), the mechanism by which oxidized mitochondrial DNA is released in the context of SLE, its relationship to NET-like structures, and its role in disease pathogenesis remain controversial.

While NETs are associated with SLE pathogenesis, this hypothesis is challenged by murine studies in which classical NETs were abolished by genetically deleting cytochrome b-245, $\beta$ polypeptide (cybb), an essential component of the NADPH oxidase complex, in the context of a lupus-prone genetic background (27). In these mice, not only was lupus still present but cybb deficiency exacerbated multiple manifestations of lupus and immune activation, including kidney disease. These results parallel prior observations in male chronic granulomatous disease (CGD) patients who carry an X-linked mutation in $c y b b$ (28), as well as their carrier mothers $(29,30)$. Furthermore, alleles of other components of the NADPH oxidase complex, neutrophil cytosolic factor $(N c f) 1$ and 2, that are thought to be less functional, are positively associated with lupus across multiple ethnic groups (31-33). Although these studies argue against the hypothesis that NETs drive lupus, it remains possible that other immune dysregulatory effects of global cybb deficiency dominantly override the requirement for NADPH oxidase-driven NETs in this case. In addition, as there have been reports that various stimuli induce distinct types of NETs, it is possible that cybb-independent NET formation could drive SLE.

To address this pivotal controversy, it is important to evaluate a second independent approach to block NET generation in the setting of SLE. Padi4 is a natural target since neutrophils from Padi4deficient mice fail to make NETs $(11,12)$. Additionally, pharmacological inhibition of Padi4 with potent and specific Padi4 inhibitors, GSK199 and GSK484, reduced NET formation in mouse and human neutrophils (15).

To investigate the role of Padi4 in lupus and nephritis, we combined genetic approaches to delete this molecule in the MRL.Fas ${ }^{p r}$ spontaneous mouse model of SLE, coupled with pharmacological inhibition of the PAD family of enzymes in 2 different IC-Fc $\gamma \mathrm{R}$-mediated nephritis models (34) to comprehensively test the contribution of Padi4-mediated processes in systemic autoimmunity and end-organ injury. We chose to eliminate Padi4 in the MRL.Fas ${ }^{l p r}$ strain, as it has been tested in both the context of cybb deficiency and PAD pharmacological inhibition $(19,20,27)$, thus allowing for direct comparison of results. The MRL.Fas ${ }^{l p r}$ model of SLE is a leading system for the study of lupus since it has the advantage of being driven by multiple genes of the MRL background coupled with spontaneous onset, as in the case of human SLE (35). Fas deficiency accelerates the disease but is not required for it, nor does it generally impact outcomes of genetic or therapeutic manipulations (35). Notably, the MRL model recapitulates nearly all American College of Rheumatology diagnostic criteria $(27,36)$ and is both type I/type II IFN dependent $(37,38)$, thereby allowing for a comprehensive analysis of the impact of Padi4 deficiency on multiple aspects of disease.

Here, we show that abrogation of Padi4 by a genetic approach and pharmacological pan-PAD inhibition did not have any impact on clinical or immunological parameters of SLE in MRL.Fas ${ }^{\text {lpr }}$ mice nor on the 2 induced nephritis models. Taken together with earlier work (27), these data challenge the concept that NETs, to the degree that NET generation relies on Padi4 $(11-16,39)$ or cybb $(4,9,40-42)$, critically drives lupus and nephritis.

\section{Results}

Padi4 deficiency has no impact on nephritis or dermatitis. To assess the role of Padi4 deficiency in SLE, we initially generated F2 cohorts of Padi4-deficient mice on the MRL.Fas ${ }^{\text {lpr }}$ background by crossing the $\mathrm{Pad4}^{-/-}$C57BL/B6 strain with Padi4 ${ }^{+/+}$MRL.Fas ${ }^{l p r}$, followed by intercrossing F1 progeny. Resulting $l p r$ homozygotes have $50 \%$ of the SLE loci and typically develop clinical features of SLE by 16 to 24 weeks of age. The F2 approach is an operationally reliable genetic screen to identify potential mediators of disease pathogenesis, an approach successfully used by our group and others $(27,43-46)$. To confirm our findings

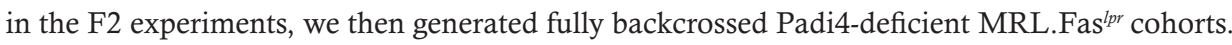



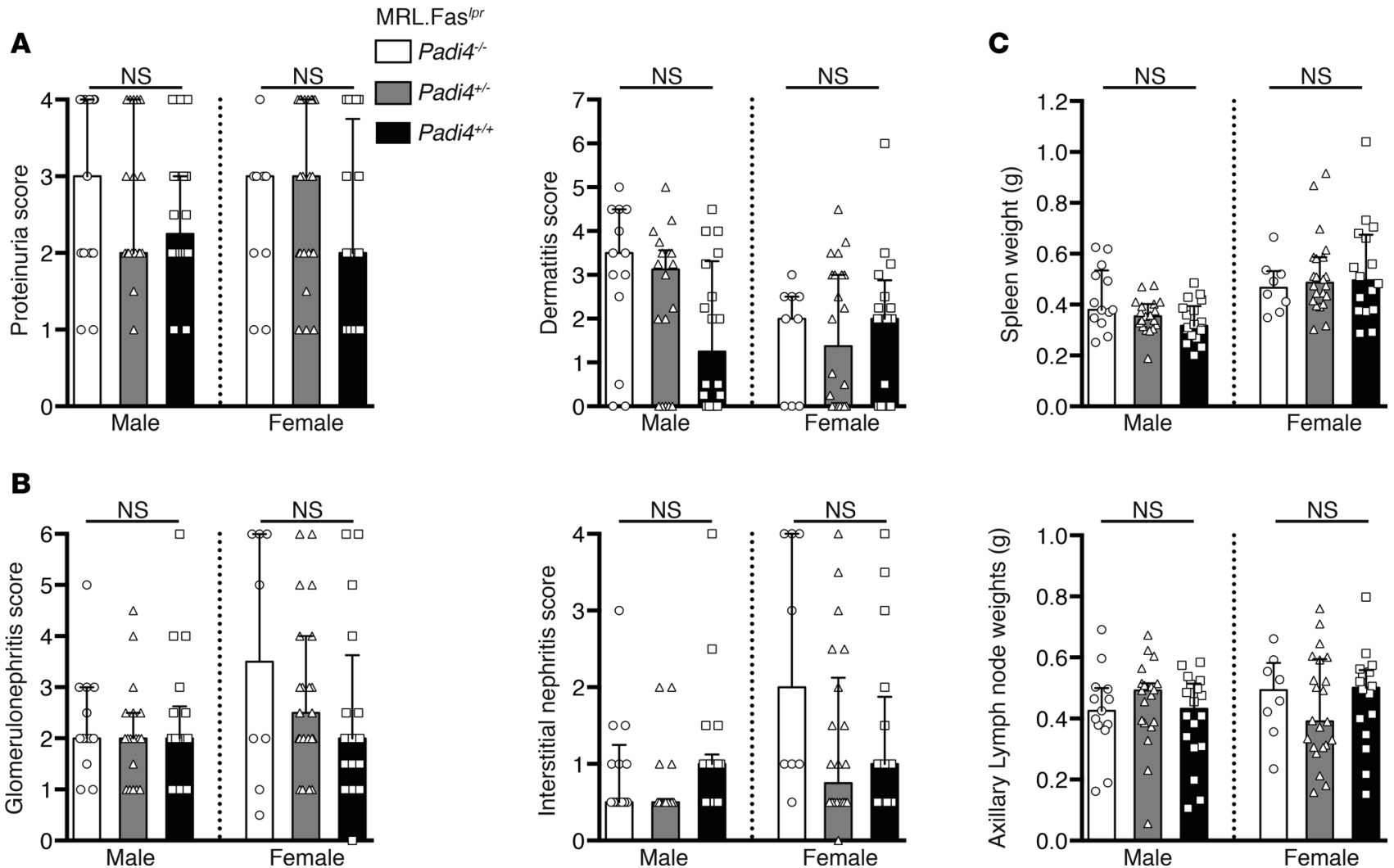

Figure 1. Padi4 genotype does not impact lupus nephritis, dermatitis, or lymphadenopathy/splenomegaly. (A) Proteinuria (left panel) and dermatitis (right panel) scores (Padi4 ${ }^{-/-}$males $n=13$; Padi4 ${ }^{+/-}$males $n=18$; Padi4 ${ }^{+/+}$males $n=18 ;$ Padi4 ${ }^{-/-}$females $n=9$; Padi4 ${ }^{+/-}$females $n=22 ;$ Padi4 ${ }^{+/+}$females $\left.n=16\right)$. (B) Glomerulonephritis (left panel) and interstitial nephritis (right panel) scores. (C) Spleen (upper panel) and axillary lymph node (lower panel) weights. Scores and weights are represented as a function of Padi4 genotype and gender at 17 weeks of age. Bars represent the median \pm interquartile range. A Kruskal-Wallis

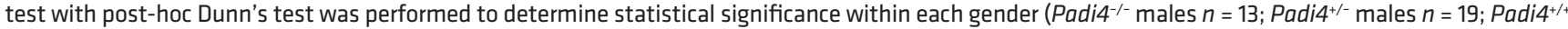
males $n=18 ;$ Padi $^{-/-}$females $n=8$; Padi4 ${ }^{+/-}$females $n=22$; Padi4 ${ }^{+/+}$females $n=16$ unless otherwise indicated). NS, not significant.

No differences in urine protein were detected in any of the F2 (Supplemental Figure 1A; supplemental material available online with this article; https://doi.org/10.1172/jci.insight.92926DS1) or fully backcrossed cohorts (Figure 1A), with the exception that Padi4 ${ }^{--}$males in the F2 cohort had more proteinuria than their Padi4-intact male counterparts (median score 2 vs. $1, P=0.028$ ). Padi4 deficiency had no impact on dermatitis in the fully backcrossed cohort (Figure 1A). No statistically significant differences in glomerulonephritis or interstitial nephritis were detected among the different Padi4 genotypes in either cohort (Figure 1B and Supplemental Figure 1B). Similarly, Padi4 genotype did not impact splenomegaly and lymphadenopathy (Figure 1C and Supplemental Figure 1C).

Padi4 genotype has no impact on loss of tolerance or anti-self responses. Padi4 genotype did not generally alter the autoantibody response or Ig titers. We did not detect any change in anti-RNA antibody (Figure 2A and Supplemental Figure 2A), anti-Sm antibody (Figure 2B and Supplemental Figure 2B), anti-nucleosome antibody (Figure 2C and Supplemental Figure 2C), rheumatoid factor (Figure 2D and Supplemental Figure 2D), or total IgM and total IgG titers (Figure 2E) in the context of Padi4 deficiency. Notably, fully backcrossed but not the F2 Padi4 $^{-1}$ males had elevated anti-Sm titers (Figure 2B). Concordant with these data, Padi4 genotype did not affect the percentage of $\mathrm{CD} 19^{\text {lo-int }} \mathrm{CD} 44^{+} \mathrm{CD} 138^{+}$intracellular $\kappa^{\text {hi }}$ antibody-forming cells (AFCs) (Figure $2 \mathrm{~F}$ and Supplemental Figure $2 \mathrm{E}$ ). Furthermore, there were no statistically significant differences in total $\kappa$ light chain AFC ELISpots (Figure 2G and Supplemental Figure 2F). Similar AFC ELISpot results were obtained for the IgG1, IgG2a, and IgM isotypes (Figure 2, H-J).

Padi4 deficiency has little impact on the myeloid and lymphoid compartments. Padi4 genotype had only a minor impact on the myeloid compartment in MRL.Fas ${ }^{p r}$ mice. The percentages of CD $11 b^{+}$Ly6G ${ }^{+}$bone marrow neutrophils and $\mathrm{CD} 11 \mathrm{~b}^{+} \mathrm{F} 4 / 80^{+} \mathrm{Gr} 1^{\text {lo-int }}$ macrophages were not statistically different among the groups in the fully backcrossed cohort (Figure 3A). Male Padi4 ${ }^{-1}$ F2s had an increased percentage 
A

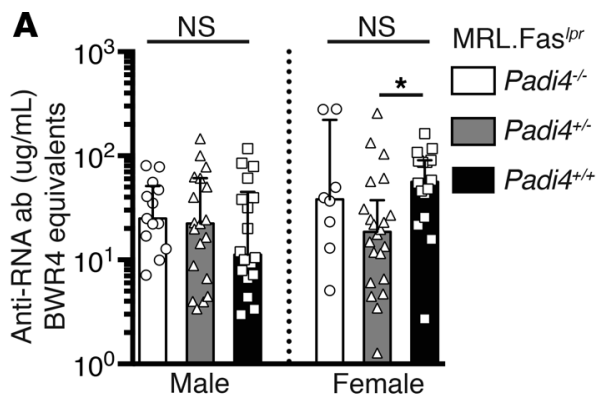

C

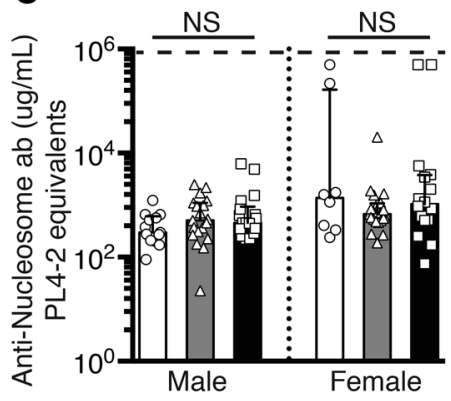

$\mathbf{F}$
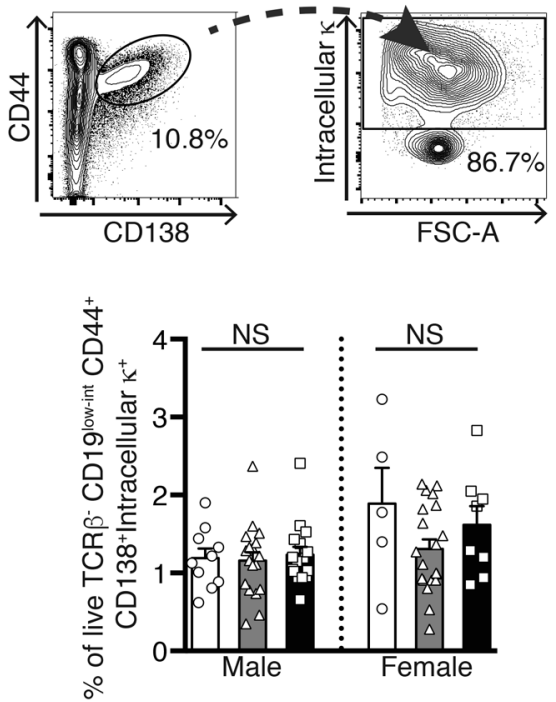

B

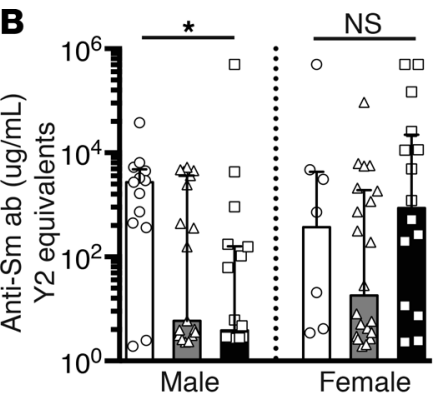

D

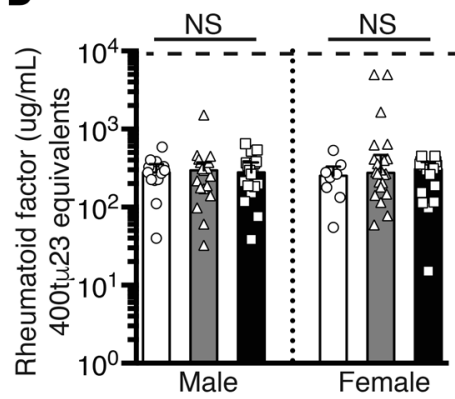

G

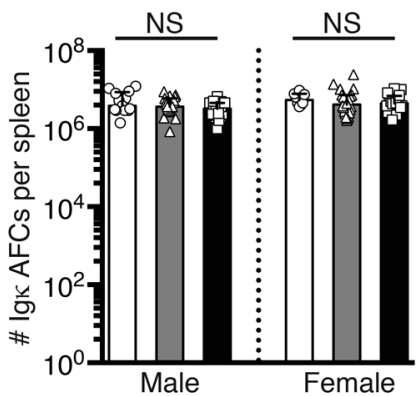

I

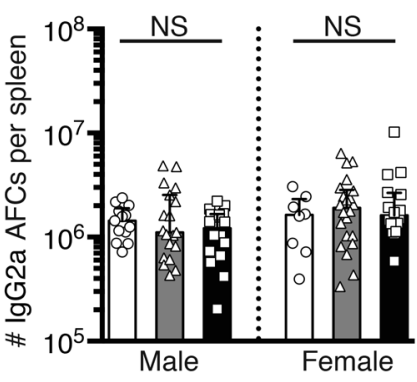

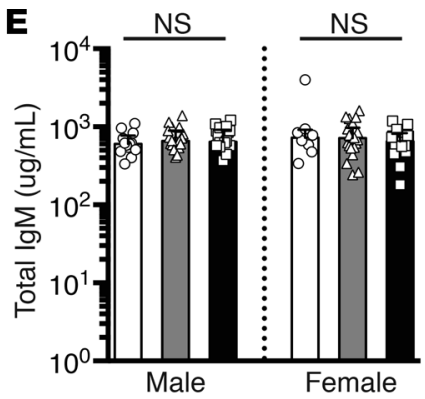
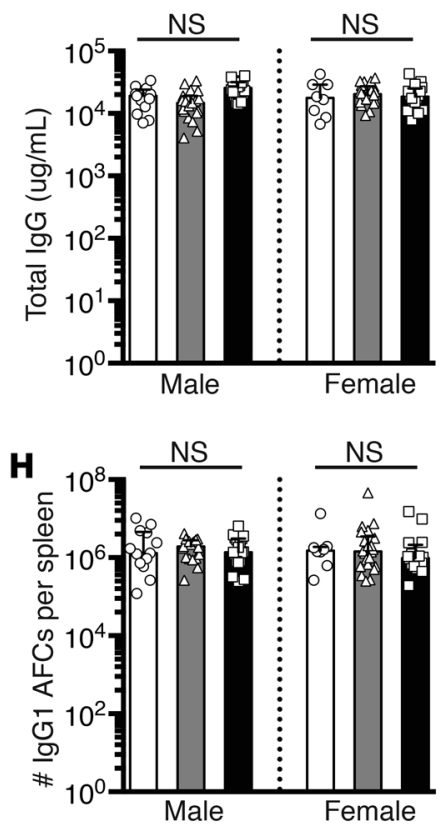

J

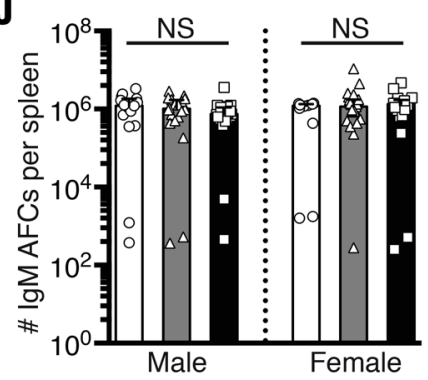

Figure 2. Padi4 genotype does not significantly alter the anti-self response or the AFC compartment. (A-D) Serum anti-RNA (A), anti-Sm (B), anti-nucleosome (C), rheumatoid factor (D), and total IgM and total IgG (E) antibody titers at 17 weeks of age. The dotted line denotes the upper limit of quantification. (F) Percentages of live cells that are TCR $\beta^{-} C^{-}$444 ${ }^{+}$CD138 $8^{+}$intracellular $\kappa^{+}$antibody-forming cells (AFCs) in spleens (Padi4 ${ }^{-/-}$males $n=10 ;$ Padi4 ${ }^{+/-}$males $n=18$; Padi4 $^{+/+}$males $n=16$; Padi4 ${ }^{-/-}$females $n=5$; Padi4 ${ }^{+/-}$females $n=20 ;$ Padi4 $^{+/+}$females $\left.n=8\right)$. (G-J) Numbers of IgK $(\mathbf{C})$, IgG1 (H), IgG2a (I), and IgM (J) AFCs per spleen as determined by ELISpot. Data representation and statistics are as in Figure 1 (Padi4 ${ }^{-/-}$males $n=13 ;$ Padi4 $^{+/-}$males $n=19$; Padi4 ${ }^{+/+}$males $n=18$; Padi4 $^{-/ \text {- }}$ females $n=8$; Padi4 ${ }^{+/-}$females $n=22$; Padi4 ${ }^{+/}$females $n=16$ unless otherwise indicated). In panel F, bar graphs are represented as the mean \pm SEM and a 1-way ANOVA with post-hoc Holm-Sidak test was performed to determine statistical significance within each gender. ${ }^{*} P<0.05$. NS, not significant.

of splenic neutrophils compared with $\mathrm{Padi4}^{+/+}$and Padi4 ${ }^{+/-}$controls, a feature not seen in the backcrossed cohort (Figure 3B and Supplemental Figure 3A). Fully backcrossed female Padi4 ${ }^{-1}$ mice had a greater percentage of splenic neutrophils compared with their heterozygous counterparts (Figure 3B). F2 Padi4 $^{-/-}$males had an increased percentage of splenic macrophages compared with Padi4 ${ }^{+/-}$males (Supplemental Figure 3A). These findings are unlikely to be biologically significant, as these data are not mirrored in both the F2 and fully backcrossed cohort and are small in magnitude. No differences in the percentage of $\mathrm{CD}^{-} 9^{-} \mathrm{CD} 11 \mathrm{c}^{+} \mathrm{MHCII}{ }^{+}$conventional DCs $(\mathrm{cDCs})$ (Figure $3 \mathrm{C}$ and Supplemental 
A

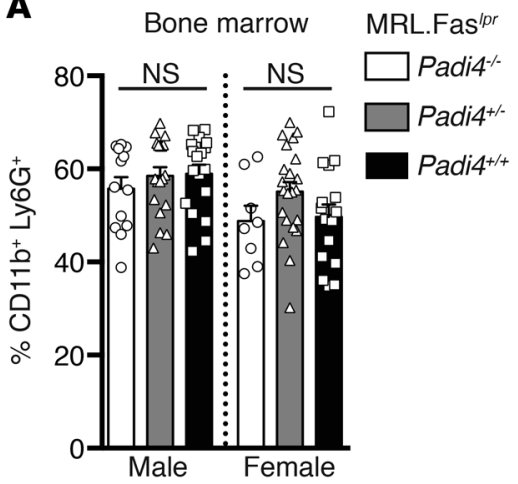

C

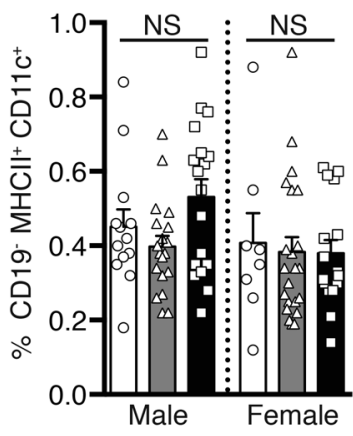

E

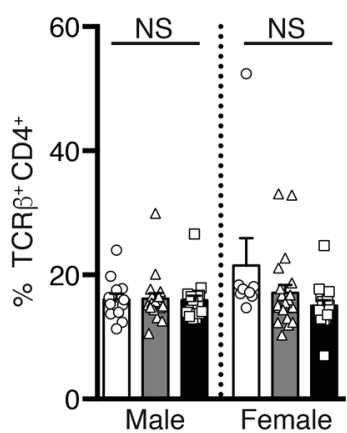

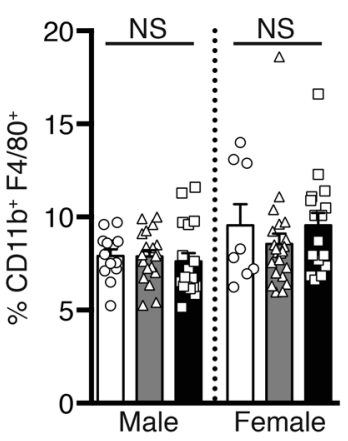
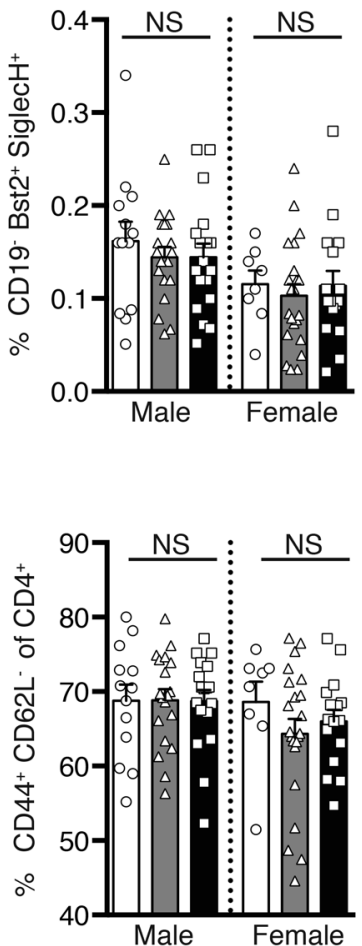
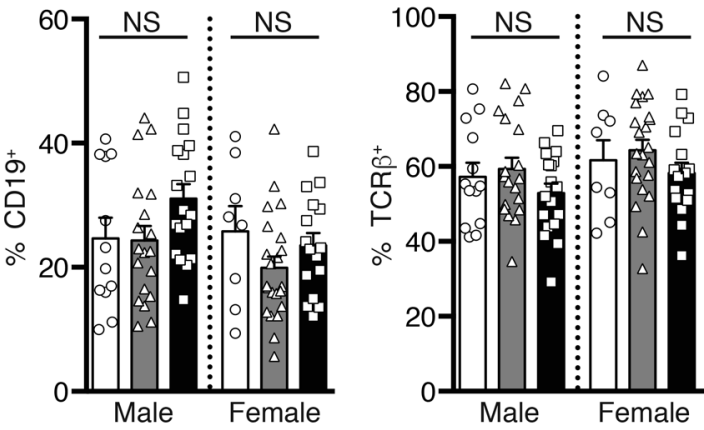

F

B
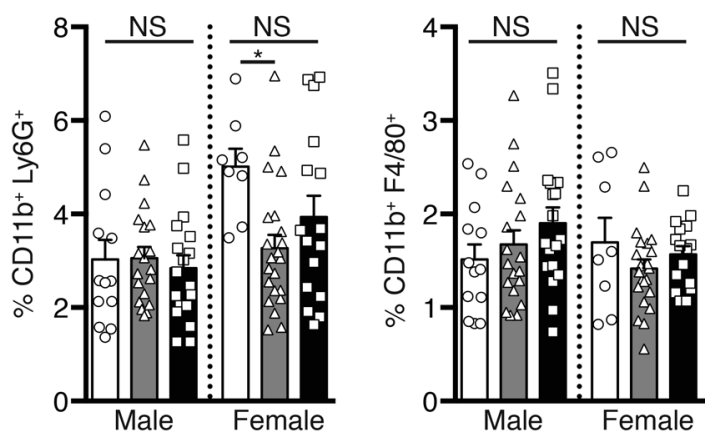

D
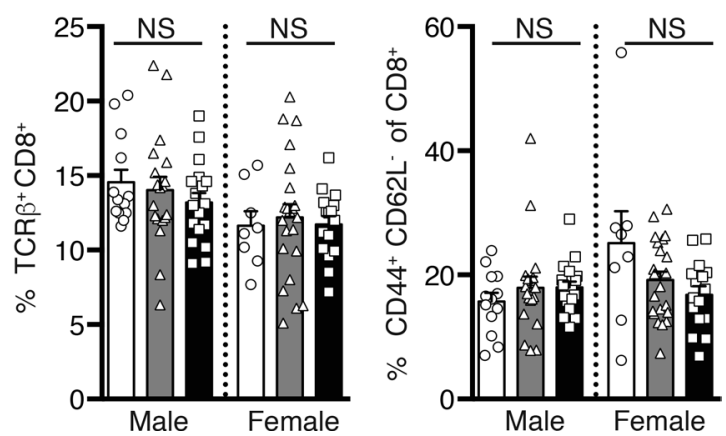

Figure 3. Padi4 genotype does not substantially affect the myeloid, DC, or T cell compartments. (A and B) Percentages of live CD11b+Ly6C+ ${ }^{+}$neutrophils (left

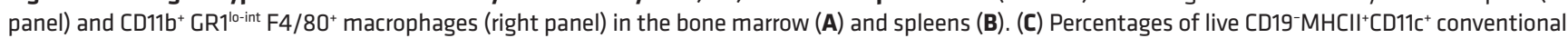
DCs (left panel) and CD19-BST2+CD11 $C^{+}$plasmacytoid DCs (right panel). (D) Percentages of live CD19+ total B cells (left panel) and TCR $\beta^{+}$total T cells (right panel). (E) Percentages of live TCR $\beta^{+} C D 4^{+} T$ cells (left panel) and of CD4+CD44+CD62L- activated T cells (right panel). (F) Percentages of live TCR $\beta^{+} C D 8^{+} T$ cells

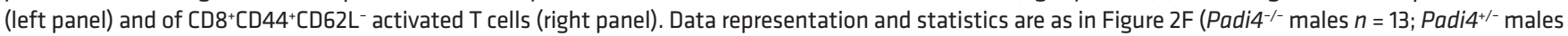
$n=19 ;$ Padi4 $^{+/+}$males $n=18$; Padi4 ${ }^{/-}$females $n=8$; Padi4 ${ }^{+/-}$females $n=22 ;$ Padi4 $^{+/+}$females $n=16$ ). NS, not significant.

Figure 3B) and CD19-BST2 ${ }^{+}$SiglecH ${ }^{+}$pDCs (Figure 3C and Supplemental Figure 3B) were identified among Padi4 genotypes in either cohort.

Padi4 deficiency did not substantially impact the lymphoid compartment. All genotypes in both cohorts exhibited indistinguishable total percentages of $\mathrm{CD} 19^{+} \mathrm{B}$ cells and TCR $\beta^{+} \mathrm{T}$ cells (Figure 3D). The percentages of $\mathrm{CD} 4^{+} \mathrm{T}$ cells and $\mathrm{CD} 4^{+} \mathrm{CD} 44^{+} \mathrm{CD} 62 \mathrm{~L}^{-}$activated $\mathrm{T}$ cells were also similar among wild-type, Padi4deficient, and heterozygous mice (Figure 3E and Supplemental Figure 3C). Similar results were obtained for naive and activated $\mathrm{CD} 8^{+} \mathrm{T}$ cells (Figure $3 \mathrm{~F}$ and Supplemental Figure 3D).

NET formation in the cremaster muscle following the reverse passive Arthus reaction is PAD4 dependent and is inhibited by Cl-Amidine. Neutrophil recognition of deposited ICs in the glomeruli can trigger FcyR-dependent inflammation leading to glomerulonephritis $(25,47,48)$. Upon stimulation of $F c \gamma R$, neutrophils are activated to produce ROS and secrete lytic enzymes that cause local tissue damage (49). We have previously 
A
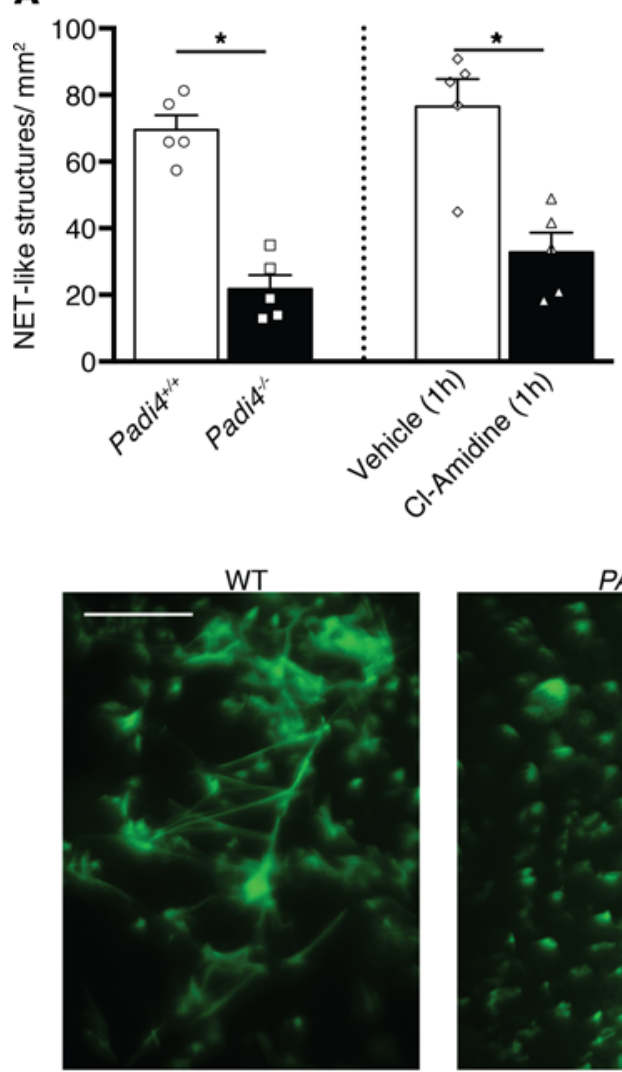

Vehicle

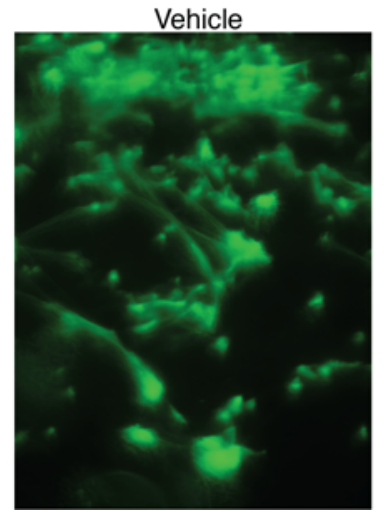

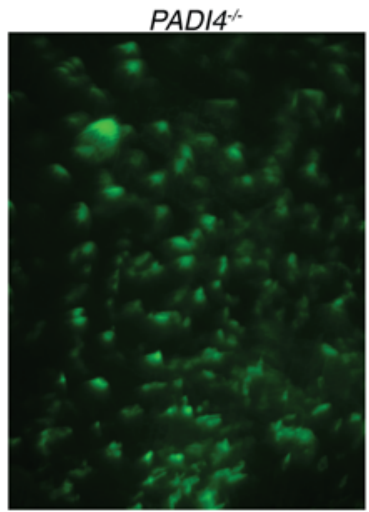

Cl-Amidine

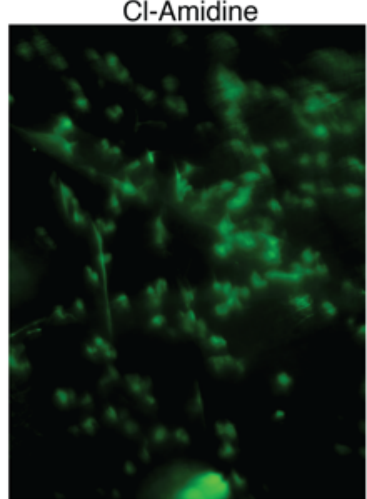

B
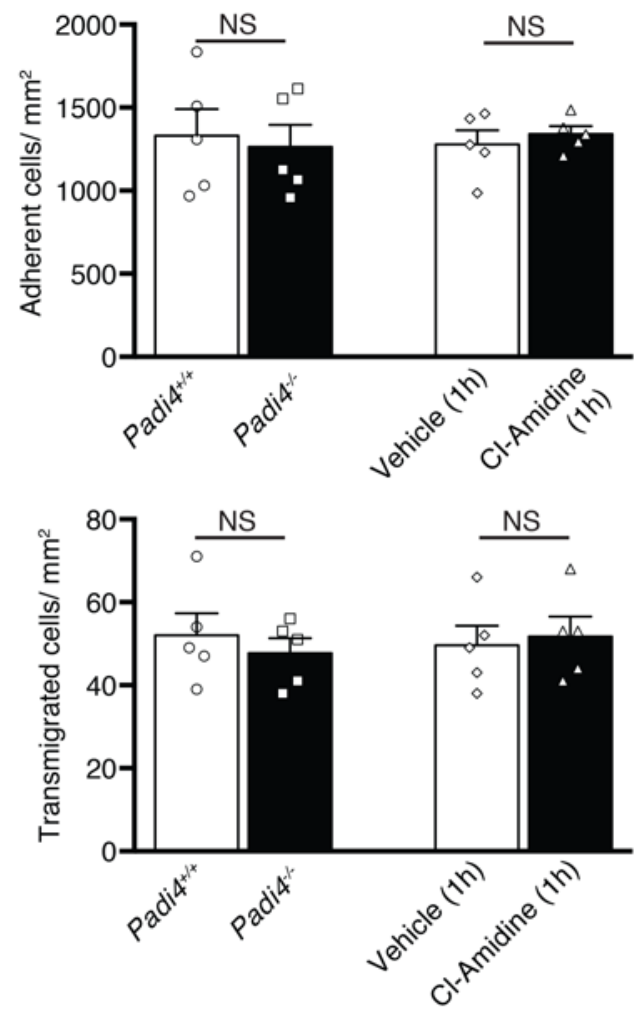

C

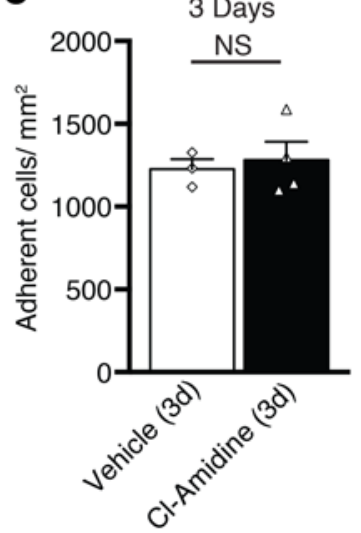

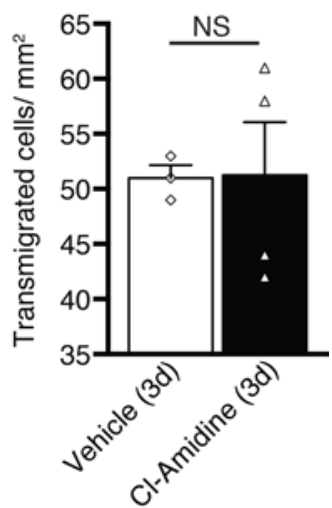

Figure 4. CI-Amidine effectively reduces NET formation in the cremaster muscle following the reverse passive Arthus (RPA) reaction. The RPA reaction was induced in the cremaster muscle. Leukocyte recruitment and neutrophil extracellular trap (NET) formation was evaluated 3 hours later. Data are represented as a function of Padi4 genotype or Cl-Amidine administration. (A) NET-like structures were visualized with Sytox green using intravital microscopy. Representative micrographs are shown. Scale bar: $100 \mu \mathrm{m}$. (B and $\mathbf{C}$ ) Number of leukocytes adherent to the vessel wall of animals in $\mathbf{A}$.

Number of transmigrated leukocytes within $75 \mu \mathrm{m}$ of each side of the vessel over $100 \mu \mathrm{m}$. Bars graphs denote the mean \pm SEM and a Welch's $t$ test was performed to determine statistical significance (A [left] and $\mathbf{B}$ vehicle and Cl-Amidine treated $n=5$ mice per group, $\mathbf{A}$ [right] and $\mathbf{C}$ vehicle $n=3$ mice per group; Cl-Amidine $n=4$ mice per group [right panel of C]). ${ }^{*} P<0.05$. NS, not significant.

shown that binding of ICs to Fc $\gamma$ Rs can also trigger the release of NETs (10). Notably, in vivo, ICs formed upon induction of the reverse passive Arthus reaction (RPA) in the cremaster muscle of the mouse induced NET formation that required neutrophil Fc $\gamma$ Rs (10).

To directly investigate the role of Padi4 in IC-Fc $\gamma \mathrm{R}$-dependent NET formation, NETs were evaluated in Padi4 $^{-1}$ mice subjected to the RPA reaction in the cremaster muscle, which results in IC deposition in the tissue that can be visualized by intravital microscopy (10). RPA-induced NET formation was significantly reduced in the Padi4 ${ }^{--}$compared with wild-type mice (Figure 4A). Likewise, treatment of mice with $N$-R-benzoyl- $N^{5}$-(2-chloro-1-iminoethyl)-L-ornithine amide (Cl-Amidine), a pan-PAD inhibitor, markedly 
A

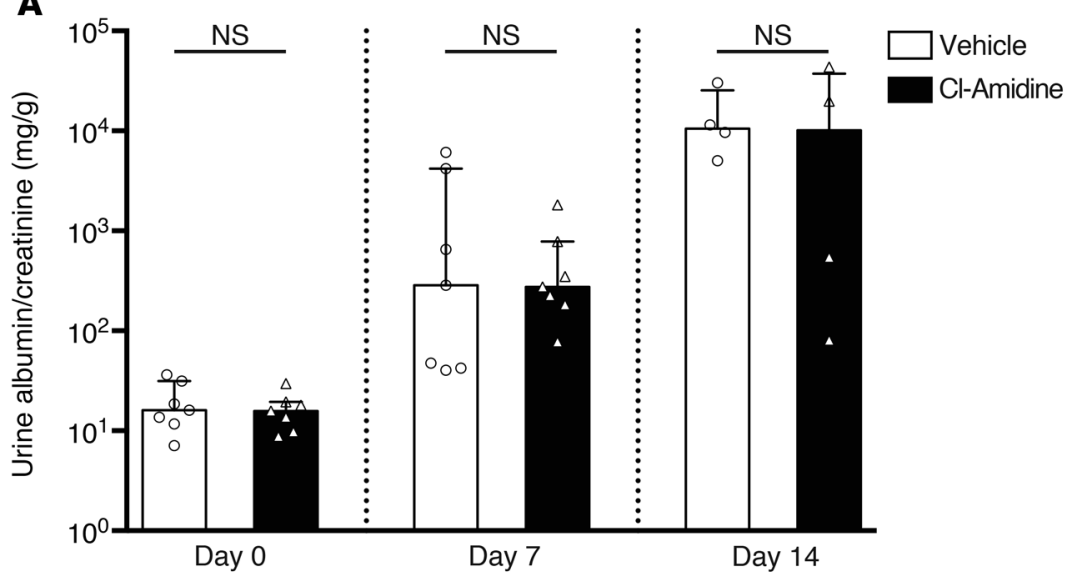

B

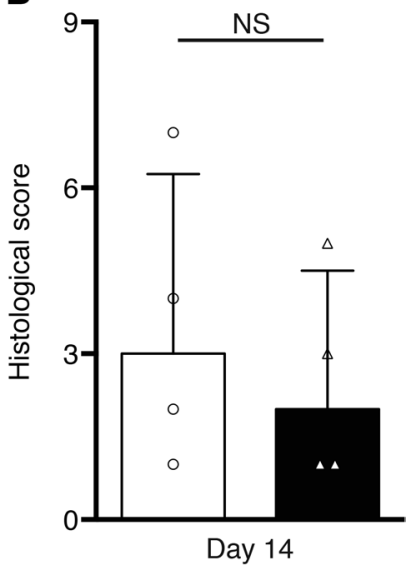

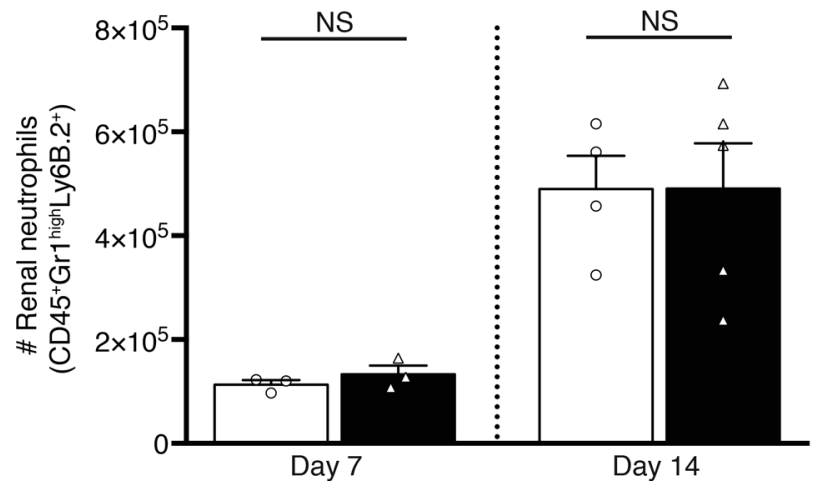

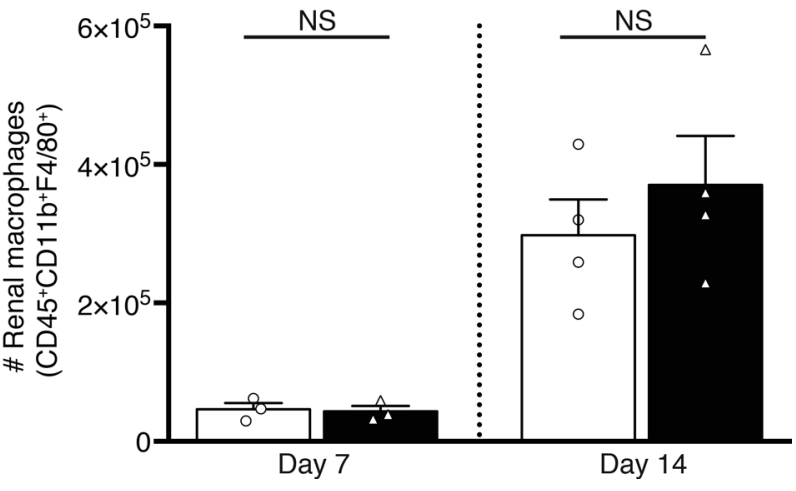

Figure 5. Cl-Amidine has no effect on kidney damage following anti-GBM nephritis. Anti-glomerular basement membrane (anti-GBM) nephritis was induced in Fc $\gamma \mathrm{RIIA} \gamma^{-1-}$ mice. (A) Albuminuria at indicated days after nephrotoxic serum injection in mice treated daily with either vehicle (PBS) or Cl-Amidine. Urine albumin was normalized to urine creatinine (day $0 n=7$; day $7 n=7$; day $14 n=4$ mice per group). (B) Kidney pathology of mice in $\mathbf{A}$ at 14 days after serum transfer. (C) Quantification of macrophages (CD45+CD11b+F4/80+) and neutrophils (CD45+ ${ }^{+} 1^{\text {hi }}$ Ly6B. $\left.2^{+}\right)$in kidneys at day $7(n=3$ mice per group) and $14(n=$ 4-5 mice per group) of mice in $\mathbf{A}$. Bars represent the median \pm interquartile range (A and $\mathbf{B})$. A Mann-Whitney $U$ test was performed to determine statistical significance. In panel C, bar graphs denote the mean \pm SEM and a Welch's $t$ test was performed to determine statistical significance. NS, not significant.

reduced NET generation to a similar degree as in Padi4 ${ }^{-/}$animals (Figure 4A), while neutrophil recruitment to the vessel wall and interstitial space was unaffected (Figure $4 \mathrm{~B}$ and Figure $4 \mathrm{C}$ ). The $\mathrm{Cl}$-Amidine was effective when given 1 hour before or for 3 consecutive days with the last dose 20 hours before the experiment (Figure 4A). These data indicate that $\mathrm{Fc} \gamma \mathrm{R}$-mediated NET generation relies on Padi4 and that Padi4 deletion or Cl-Amidine treatment effectively reduces Fc $\gamma$ R-dependent NET formation in vivo without affecting neutrophil recruitment.

Inhibition of PADs has no effect on kidney damage following anti-GBM nephritis. To independently confirm our genetic results that Padi4 deficiency did not affect nephritis in the MRL.Fas ${ }^{\text {lpr }}$ lupus model, we tested Cl-Amidine in a murine model of proliferative anti-glomerular basement membrane (anti-GBM) nephritis induced by the injection of rabbit anti-GBM-containing sera in mice expressing Fc $\gamma$ RIIA selectively on neutrophils (48). In this model, antibodies directed against the GBM trigger an inflammatory reaction that results in severe damage of the kidney that depends on neutrophil Fc $\gamma$ RIIA. Daily treatment of mice subjected to the anti-GBM nephritis model with Cl-Amidine did not inhibit the development of kidney damage, as measured by proteinuria (Figure 5A) and histological signs of inflammation (Figure 5B). Further analysis of leukocyte recruitment to the kidneys showed no reduction in $\mathrm{Cl}$-Amidine-treated versus vehicle-treated animals (Figure 5C).

PAD inhibition does not ameliorate kidney damage in a passive human SLE serum-transfer model of lupus nephritis. To further evaluate the effects of PAD inhibition specifically in the development of organ damage relevant to 
A

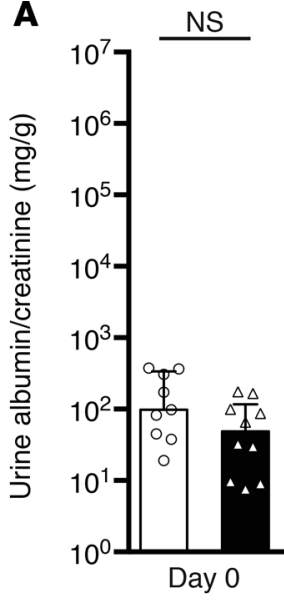

NS

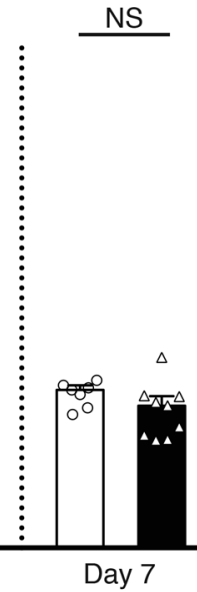

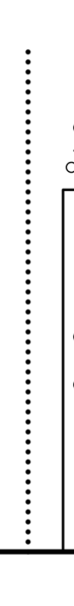

NS
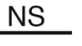

$\vdots$

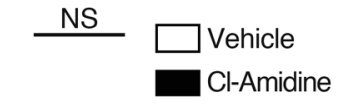

NS

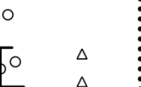

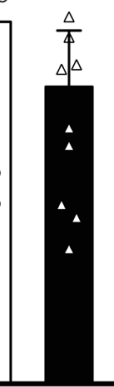

Day 10

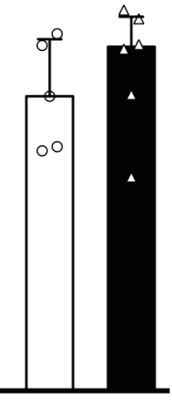

Day 14
B

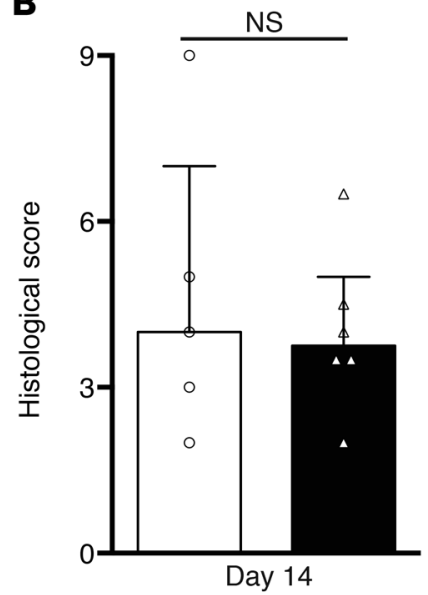

C
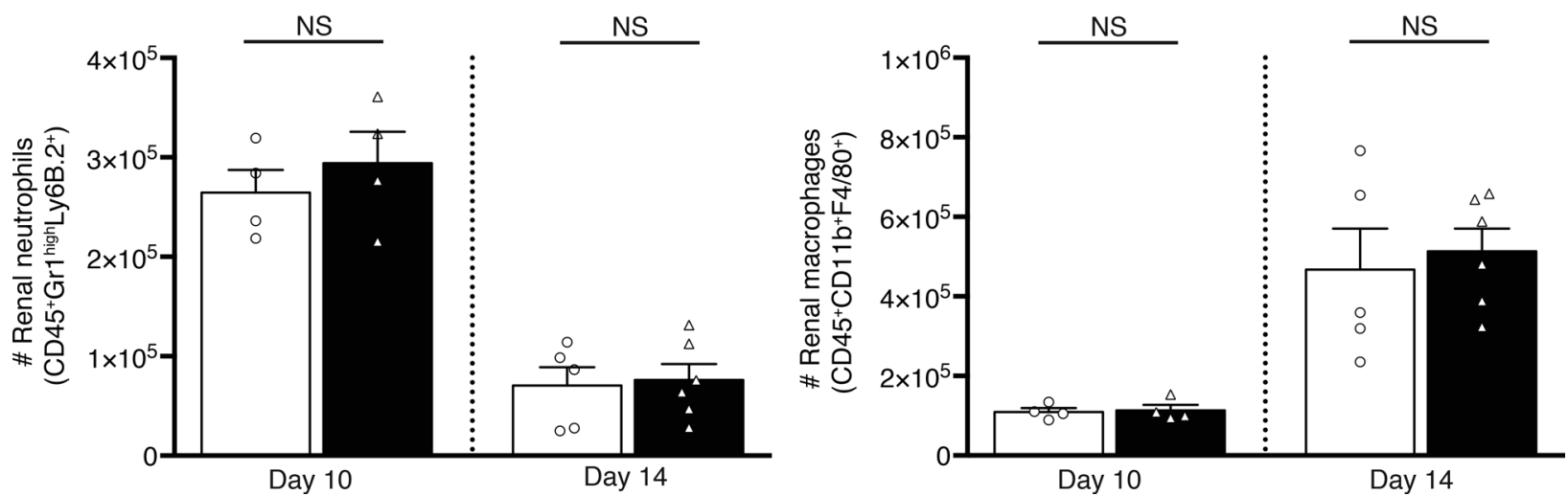

Figure 6. CI-Amidine does not ameliorate kidney damage in the SLE serum-transfer model. Nephritis was induced by the intravenous injection of systemic lupus erythematosus (SLE) serum into Fc $\gamma \mathrm{RIIA} \gamma^{-1-}$ Mac-1-1- mice. (A) Albuminuria at the indicated days after SLE sera injection in mice treated daily with either vehicle (PBS) or Cl-Amidine. Urine albumin was normalized to urine creatinine (day 0 vehicle $n=9, \mathrm{Cl}$-Amidine $n=10$; day 7 vehicle $n=$ 7, Cl-Amidine $n=9$; day 10 vehicle $n=10$, Cl-Amidine $n=10$; day 14 vehicle $n=5$, Cl-Amidine $n=6$ mice per group). (B) Kidney pathology of mice in (A)

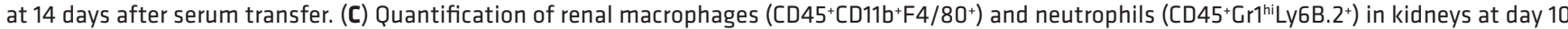
( $n=4$ mice per group) and 14 (vehicle $n=5$; Cl-Amidine $n=6$ mice per group) of mice in $\mathbf{A}$. Bars represent the median \pm interquartile range (A and $\mathbf{B}$ ). A Mann-Whitney $U$ test was performed to determine statistical significance. In panel C, bars graphs denote the mean \pm SEM and a Welch's $t$ test was performed to determine statistical significance. NS, not significant.

human disease, we used a passive human SLE serum-transfer model of nephritis (34). In this model, mice that transgenically express the human Fc $\gamma$ RIIA selectively on neutrophils and additionally lack the $\beta 2$ integrin Mac-1 are susceptible to developing nephritis upon the i.v. injection of serum from patients with SLE (34). At day 10 after sera injection such mice develop proteinuria that correlates with neutrophil infiltration into the kidneys. Histological signs of disease including hypercellularity, endocapillary proliferation, and crescent formation are evident at day 14. Daily i.p. treatment with $\mathrm{Cl}$-Amidine did not reduce albuminuria (Figure 6A) or histological signs of kidney damage (Figure 6B). Moreover, we did not observe a reduction in neutrophil or macrophage infiltration compared with vehicle-treated animals (Figure 6C).

\section{Discussion}

Collectively, these genetic and inhibitor studies in multiple relevant models fail to support the hypothesis that targeting of Padi4, and by extension Padi4-dependent NET generation, alters immune system composition or pathology in SLE. Execution of 2 independent genetic experiments using a mixed background F2 and a fully backcrossed Padi4-deficient MRL.Fas ${ }^{p r}$ cohort, with both yielding the same results, strengthens these conclusions. Furthermore, Fc $\gamma$ Rs play a key role in autoimmune-mediated organ damage $(50,51)$. We demonstrate that pharmacological inhibition of PADs using Cl-Amidine had no effect 
on neutrophil recruitment or glomerular injury in 2 independent models of IC-Fc $\gamma$ R-mediated glomerulonephritis. Moreover, corroborating these studies, Padi4 deficiency does not reduce renal disease or the anti-self response in the pristane model of SLE (52).

Our Padi4-deficient MRL.Fas ${ }^{p r}$ results are discordant with previously reported PAD inhibitor studies utilizing Cl-Amidine and BB-Cl-Amidine in both the MRL.Fas ${ }^{p r}(19)$ and New Zealand mixed 2328 (NZM) (20) models. One possible explanation is that the (halo) acetamidine-based PAD inhibitors could affect other PAD family members $(19,53)$. As with most inhibitors administered at high dose over long periods in vivo, it is difficult to exclude off-target effects even beyond the related PAD enzymes. Despite the fact that only Padi4 has been implicated in NET formation and its deletion demonstrates a nonredundant effect on NET assays $(11,12,15)$, it can be speculated that the ability of (halo) acetamidine-based PAD inhibitors to block related enzymes could account for the differences between specific genetic deletion of Padi4 and prior inhibitor studies. However, we believe this explanation to be less likely as Cl-Amidine pan-PAD specificity would not account for the lack of phenotype identified in the inhibitor studies using inducible models of glomerulonephritis reported here.

Moreover, our inhibitor data in IC-Fc $\gamma \mathrm{R}$-dependent nephritis models conflict with a recent report by Ander's group demonstrating that $\mathrm{Cl}$-Amidine reduces severity of proliferative nephritis in the anti-GBM model (54). The discrepancies with published data are unlikely due to differences in treatment protocols or pharmacokinetic issues. As our studies were conducted in multiple models and in various mouse strains housed at 3 different animal facilities, it is unlikely that the lack of observed phenotype is due to differences in the microbiome. Furthermore, disease in MRL.Fas ${ }^{\text {lpr }}$ germ-free mice was not grossly altered (55), again suggesting that the microbiome is not a major factor in this model.

Although Cl-Amidine has a short half-life, it is an irreversible inhibitor, allowing for prolonged inhibition of NET generation (56). We confirmed Cl-Amidine efficacy in vivo by visualizing NETs in the cremaster muscle following the RPA reaction by intravital microscopy. Cl-Amidine continued to significantly reduce NET formation to levels observed in Padi4-deficient mice (Figure 4A) 20 hours after administration, reinforcing the conclusion that the protocol of $\mathrm{Cl}$-Amidine treatment in our nephritis model was effective in reducing PAD activity. Since PAD inhibition reduces NET formation to that observed in Padi4-deficient mice, it is unlikely that other PAD family members are serving in a compensatory capacity to generate NETs.

Neutrophils exposed to lupus serum and SLE-associated stimuli have a greater propensity to generate NETs $(18,20,34)$. However, our data disassociate the correlation between NET formation in lupus patients and mice from disease pathogenesis. Close analysis of published data in clinical settings is consistent with this dissociation. For instance, while NET degradation is impaired in some SLE patients, in fact 59\%-88\% of SLE patients actually do degrade NETs adequately $(21,22,57)$. Additionally, no statistical correlation was found between autoantibody titers, therapy, SLEDAI, and ex vivo NET production at baseline (17). Taken together, the data reported here coupled with our previously published results in cybb-deficient lupus-prone mice should lead the field to revisit and question whether NETs per se are critical drivers of autoimmunity or end-organ damage.

Further, an important contribution of the current work is that it reveals a distinction between Padi4and cybb-deficient lupus-prone mice, in that only the latter get exacerbated disease. As cybb and Padi4 operate at distinct phases of NET-formation pathways, it remains possible that the aberrant products of presumably frustrated NET generation that would occur in the absence of each required protein might differ, in turn accounting for the disparate disease phenotypes. As cybb deficiency leads to markedly exacerbated disease, the products of neutrophil death in the absence of cybb could be uniquely immunogenic. Alternatively, though not exclusively, cybb deficiency might promote disease owing to lack of a fundamental regulatory activity of cybb. Indeed, there are multiple reported means by which NADPH oxidase can inhibit inflammation $(58,59)$, including neutrophil- $(60,61)$, macrophage- $(62)$, and lymphocyte-dependent $(63,64)$ mechanisms. Recently, cybb function was found to be required for LC3-associated phagocytosis (LAP) (65). LAP has been linked to the concurrent production of suppressive rather than proinflammatory cytokines, and mice incapable of performing LAP develop autoimmunity (66).

NETs are evolutionarily conserved, suggesting a homeostatic function (67). Consistent with the modified view favored by our results, other recent publications suggest that NETs are not always proinflammatory and may even be immunomodulatory. Clearance of NET-like structures is an immunologically silent process and does not lead to proinflammatory cytokine production (68). Mice immunized with NETs do not break tolerance and show trace urinary protein levels up to 14 weeks after immunization (69). Moreover, aggregated NETs can ameliorate inflammation by inducing proteolytic cleavage of inflammatory mediators 
(61). NETs thus may have evolved, in part, to protect us from rather than promote autoimmunity (70).

\section{Methods}

Detailed supplemental methods are available online.

Mice. Padi4-deficient C57BL/6 mice were a gift from Yanming Wang (Center for Eukaryotic Gene Regulation, Department of Biochemistry and Molecular Biology, Pennsylvania State University, University Park, Pennsylvania, USA). F1 offspring were generated by crossing Padi4-deficient mice on the C57BL/6 background, with the MRL.Fas ${ }^{\text {pr }}$ strain (The Jackson Laboratory, catalog 000485). Offspring were then intercrossed to generate an F2 cohort. F2 Fas ${ }^{l p r}$ homozygotes were analyzed for disease pathology as described below. Fully backcrossed mice were obtained by crossing the Padi4 ${ }^{--}$allele onto the MRL. Fas $^{\text {lpr }}$ background for 9 generations. Heterozygous mice were then intercrossed to produce an experimental cohort. SLE pathology was analyzed at 16 weeks for F2 and 17 weeks for backcrossed cohorts.

Fc $\gamma$ RIIA-expressing, $\gamma$-chain-deficient $\left(\mathrm{Fc}_{\mathrm{R}} \mathrm{RIIA}^{+} / \gamma^{-/}\right)$mice and Fc $\gamma$ RIIA-expressing, $\gamma$-chain-deficient, and Mac1-deficient (Fc $\left.\gamma \mathrm{RIIA}{ }^{+} / \gamma^{-/-} / \mathrm{Mac}^{-/-}\right)$mice were generated as previously described $(34,48)$. Wild-type C57BL/6 were obtained from Jackson Laboratories (catalog 000664).

All mice were housed under specific pathogen-free (SPF) conditions.

Evaluation of SLE pathology. MRL.Fas ${ }^{l p r}$ SLE cohorts were analyzed as previously described $(27,37)$.

$R P A$ reaction in the cremaster muscle. The RPA reaction in the cremaster was performed as described previously (10). In experiments using Cl-Amidine (Millipore), mice received $10 \mathrm{mg} / \mathrm{kg}$ Cl-Amidine or vehicle control via i.p. injection either 1 hour before the experiment or for 3 consecutive days with the last dose 20 hours before the experiment (20).

Serum-transfer SLE nephritis. Nephritis was induced using serum from SLE patients as previously described (34). Mice received either vehicle control (PBS) or Cl-Amidine at $10 \mathrm{mg} / \mathrm{kg} / \mathrm{d}$ i.p. (20) for the duration of the experiment.

Anti-GBM nephritis. Experimental anti-GBM nephritis was induced as previously reported (48). Mice received either vehicle control (PBS) or Cl-Amidine at $10 \mathrm{mg} / \mathrm{kg} / \mathrm{d}$ (20) i.p. for the duration of the experiment.

Statistics. Statistical analysis was performed using Prism 6.0 (GraphPad). A Kruskal-Wallis test with post-hoc Dunn's test, 2-tailed Mann-Whitney $U$ test, 2-tailed Welch's $t$ test, and a 1-way ANOVA with post hoc Holm-Sidak test were performed where indicated and appropriate. A $P$ value less than 0.05 was considered statistically significant.

Study approval. Animal studies were approved by (a) the University of Pittsburgh IACUC, (b) Yale University's IACUC, or (c) the Harvard Medical School Animal Care and Use Committee.

\section{Author contributions}

RG, JH, FR, AC, and AM performed experiments and analyzed data. HN, MK, and SB performed histopathological evaluation of the kidneys. RG, JH, FR, KN, TNM, and MS designed experiments and wrote the manuscript.

\section{Acknowledgments}

This study utilized the NIH sponsored Pittsburgh Center for Kidney Research. This work was supported by the Lupus Insight Prize from the Alliance for Lupus Research, Lupus Foundation of America, and Lupus Research Institute (to MS), NIH R01-AR044077 (to MS), NIH T32-AI08944303 (to RG), NIH T32GM008208-22 (to RG), NIH Ruth L Kirschstein F30-DK091993-02 (to AC), NIH-2T32GM07205 (to AC), NIH R01-HL065095 (to TNM), and the Else Kröner-Fresenius-Stiftung 2014_A80 (to JH).

Address correspondence to: Mark J. Shlomchik, W1052 Biomedical Science Tower 200 Lothrop Street, Pittsburgh, Pennsylvania 15261, USA. Phone: 412.648.8771; E-mail: mshlomch@pitt.edu.

JMH's present address is: Department of Anesthesiology, Intensive Care Medicine and Pain Therapy, University Hospital Muenster, Muenster, Germany.

FR's present address is: Departamento de Inmunología y Reumatología, Instituto Nacional de Ciencias Médicas y Nutrición Salvador Zubirán, Mexico City, Mexico. 
1. Lisnevskaia L, Murphy G, Isenberg D. Systemic lupus erythematosus. Lancet. 2014;384(9957):1878-1888.

2. Mahajan A, Herrmann M, Muñoz LE. Clearance deficiency and cell death pathways: a model for the pathogenesis of SLE. Front Immunol. 2016;7:35.

3. Brinkmann V, et al. Neutrophil extracellular traps kill bacteria. Science. 2004;303(5663):1532-1535.

4. Fuchs TA, et al. Novel cell death program leads to neutrophil extracellular traps. J Cell Biol. 2007;176(2):231-241.

5. Yipp BG, Kubes P. NETosis: how vital is it? Blood. 2013;122(16):2784-2794.

6. Yousefi S, Mihalache C, Kozlowski E, Schmid I, Simon HU. Viable neutrophils release mitochondrial DNA to form neutrophil extracellular traps. Cell Death Differ. 2009;16(11):1438-1444.

7. Caielli S, et al. Oxidized mitochondrial nucleoids released by neutrophils drive type I interferon production in human lupus. $J$ Exp Med. 2016;213(5):697-713.

8. Lood C, et al. Neutrophil extracellular traps enriched in oxidized mitochondrial DNA are interferogenic and contribute to lupus-like disease. Nat Med. 2016;22(2):146-153.

9. Remijsen Q, et al. Neutrophil extracellular trap cell death requires both autophagy and superoxide generation. Cell Res. 2011;21(2):290-304.

10. Chen K, et al. Endocytosis of soluble immune complexes leads to their clearance by Fc $\gamma$ RIIIB but induces neutrophil extracel lular traps via Fc $\gamma$ RIIA in vivo. Blood. 2012;120(22):4421-4431.

11. Li P, Li M, Lindberg MR, Kennett MJ, Xiong N, Wang Y. PAD4 is essential for antibacterial innate immunity mediated by neutrophil extracellular traps. J Exp Med. 2010;207(9):1853-1862.

12. Hemmers S, Teijaro JR, Arandjelovic S, Mowen KA. PAD4-mediated neutrophil extracellular trap formation is not required for immunity against influenza infection. PLoS One. 2011;6(7):e22043.

13. Wang Y, et al. Histone hypercitrullination mediates chromatin decondensation and neutrophil extracellular trap formation. $J$ Cell Biol. 2009;184(2):205-213.

14. Martinod K, et al. Neutrophil histone modification by peptidylarginine deiminase 4 is critical for deep vein thrombosis in mice. Proc Natl Acad Sci USA. 2013;110(21):8674-8679.

15. Lewis HD, et al. Inhibition of PAD4 activity is sufficient to disrupt mouse and human NET formation. Nat Chem Biol. 2015;11(3):189-191.

16. Kolaczkowska E, et al. Molecular mechanisms of NET formation and degradation revealed by intravital imaging in the liver vasculature. Nat Commun. 2015;6:6673.

17. Villanueva E, et al. Netting neutrophils induce endothelial damage, infiltrate tissues, and expose immunostimulatory molecules in systemic lupus erythematosus. J Immunol. 2011;187(1):538-552.

18. Garcia-Romo GS, et al. Netting neutrophils are major inducers of type I IFN production in pediatric systemic lupus erythematosus. Sci Transl Med. 2011;3(73):73ra20.

19. Knight JS, et al. Peptidylarginine deiminase inhibition disrupts NET formation and protects against kidney, skin and vascular disease in lupus-prone MRL/lpr mice. Ann Rheum Dis. 2015;74(12):2199-2206.

20. Knight JS, et al. Peptidylarginine deiminase inhibition is immunomodulatory and vasculoprotective in murine lupus. J Clin Invest. 2013;123(7):2981-2993.

21. Hakkim A, et al. Impairment of neutrophil extracellular trap degradation is associated with lupus nephritis. Proc Natl Acad Sci USA. 2010;107(21):9813-9818.

22. Leffler J, et al. Neutrophil extracellular traps that are not degraded in systemic lupus erythematosus activate complement exacerbating the disease. J Immunol. 2012;188(7):3522-3531.

23. Denny MF, et al. A distinct subset of proinflammatory neutrophils isolated from patients with systemic lupus erythematosus induces vascular damage and synthesizes type I IFNs. J Immunol. 2010;184(6):3284-3297.

24. Lande R, et al. Neutrophils activate plasmacytoid dendritic cells by releasing self-DNA-peptide complexes in systemic lupus erythematosus. Sci Transl Med. 2011;3(73):73ra19.

25. Clynes R, Dumitru C, Ravetch JV. Uncoupling of immune complex formation and kidney damage in autoimmune glomerulonephritis. Science. 1998;279(5353):1052-1054.

26. Tsuboi N, Asano K, Lauterbach M, Mayadas TN. Human neutrophil Fcgamma receptors initiate and play specialized nonredundant roles in antibody-mediated inflammatory diseases. Immunity. 2008;28(6):833-846.

27. Campbell AM, Kashgarian M, Shlomchik MJ. NADPH oxidase inhibits the pathogenesis of systemic lupus erythematosus. Sci Transl Med. 2012;4(157):157ra141.

28. Winkelstein JA, et al. Chronic granulomatous disease. Report on a national registry of 368 patients. Medicine (Baltimore). 2000;79(3):155-169.

29. Schaller J. Illness resembling lupus erythematosus in mothers of boys with chronic granulomatous disease. Ann Intern Med. 1972;76(5):747-750

30. Cale CM, Morton L, Goldblatt D. Cutaneous and other lupus-like symptoms in carriers of X-linked chronic granulomatous disease: incidence and autoimmune serology. Clin Exp Immunol. 2007;148(1):79-84.

31. Jacob CO, et al. Lupus-associated causal mutation in neutrophil cytosolic factor 2 (NCF2) brings unique insights to the structure and function of NADPH oxidase. Proc Natl Acad Sci USA. 2012;109(2):E59-E67.

32. Kim-Howard X, et al. Allelic heterogeneity in NCF2 associated with systemic lupus erythematosus (SLE) susceptibility across four ethnic populations. Hum Mol Genet. 2014;23(6):1656-1668.

33. Zhao J, et al. A missense variant in NCF1 is associated with susceptibility to multiple autoimmune diseases. Nat Genet. 2017;49(3):433-437.

34. Rosetti F, et al. Human lupus serum induces neutrophil-mediated organ damage in mice that is enabled by Mac-1 deficiency. $J$ Immunol. 2012;189(7):3714-3723.

35. Nickerson KM, Shlomchik MJ. Animal models of autoimmunity. In: Ratcliffe MJH, ed. Encyclopedia of Immunobiology. Oxford: Academic Press; 2016:227-240.

36. Hochberg MC. Updating the American College of Rheumatology revised criteria for the classification of systemic lupus erythematosus. Arthritis Rheum. 1997;40(9):1725. 
37. Nickerson KM, Cullen JL, Kashgarian M, Shlomchik MJ. Exacerbated autoimmunity in the absence of TLR9 in MRL.Fas(lpr) mice depends on Ifnar1. J Immunol. 2013;190(8):3889-3894.

38. Peng SL, Moslehi J, Craft J. Roles of interferon-gamma and interleukin-4 in murine lupus. J Clin Invest. 1997;99(8):1936-1946.

39. Rohrbach AS, Slade DJ, Thompson PR, Mowen KA. Activation of PAD4 in NET formation. Front Immunol. $2012 ; 3: 360$.

40. Bianchi M, et al. Restoration of NET formation by gene therapy in CGD controls aspergillosis. Blood. 2009;114(13):2619-2622.

41. Hakkim A, et al. Activation of the Raf-MEK-ERK pathway is required for neutrophil extracellular trap formation. Nat Chem Biol. 2011;7(2):75-77.

42. Almyroudis NG, Grimm MJ, Davidson BA, Röhm M, Urban CF, Segal BH. NETosis and NADPH oxidase: at the intersection of host defense, inflammation, and injury. Front Immunol. 2013;4:45.

43. Christensen SR, Kashgarian M, Alexopoulou L, Flavell RA, Akira S, Shlomchik MJ. Toll-like receptor 9 controls anti-DNA autoantibody production in murine lupus. J Exp Med. 2005;202(2):321-331.

44. Shlomchik MJ, Madaio MP, Ni D, Trounstein M, Huszar D. The role of B cells in 1pr/lpr-induced autoimmunity. J Exp Med. 1994;180(4):1295-1306.

45. Ma J, et al. Autoimmune lpr/lpr mice deficient in CD40 ligand: spontaneous Ig class switching with dichotomy of autoantibody responses. J Immunol. 1996;157(1):417-426.

46. Sharma S, et al. Suppression of systemic autoimmunity by the innate immune adaptor STING. Proc Natl Acad Sci USA. 2015;112(7):E710-E717.

47. Suzuki Y, et al. Distinct contribution of $F_{C}$ receptors and angiotensin II-dependent pathways in anti-GBM glomerulonephritis Kidney Int. 1998;54(4):1166-1174.

48. Tsuboi N, Asano K, Lauterbach M, Mayadas TN. Human neutrophil Fcgamma receptors initiate and play specialized nonredundant roles in antibody-mediated inflammatory diseases. Immunity. 2008;28(6):833-846

49. Mayadas TN, Tsokos GC, Tsuboi N. Mechanisms of immune complex-mediated neutrophil recruitment and tissue injury. Circulation. 2009;120(20):2012-2024.

50. Hargreaves CE, et al. Fcy receptors: genetic variation, function, and disease. Immunol Rev. 2015;268(1):6-24.

51. Takai T. Fc receptors and their role in immune regulation and autoimmunity. J Clin Immunol. 2005;25(1):1-18.

52. Kienhöfer D, et al. Experimental lupus is aggravated in mouse strains with impaired induction of neutrophil extracellular traps. JCI Insight. 2017;2(10):92920. doi:10.1172/jci.insight.92920.

53. Knuckley B, et al. Substrate specificity and kinetic studies of PADs 1, 3, and 4 identify potent and selective inhibitors of protein arginine deiminase 3. Biochemistry. 2010;49(23):4852-4863.

54. Kumar SV, et al. Neutrophil extracellular trap-related extracellular histones cause vascular necrosis in severe GN. $J$ Am Soc Nephrol. 2015;26(10):2399-2413.

55. Maldonado MA, et al. The role of environmental antigens in the spontaneous development of autoimmunity in MRL-lpr mice. J Immunol. 1999;162(11):6322-6330.

56. Luo Y, et al. Inhibitors and inactivators of protein arginine deiminase 4: functional and structural characterization. Biochemistry. 2006;45(39):11727-11736.

57. Leffler J, et al. Degradation of neutrophil extracellular traps co-varies with disease activity in patients with systemic lupus erythematosus. Arthritis Res Ther. 2013;15(4):R84.

58. Lee K, Won HY, Bae MA, Hong JH, Hwang ES. Spontaneous and aging-dependent development of arthritis in NADPH oxidase 2 deficiency through altered differentiation of CD11b+ and Th/Treg cells. Proc Natl Acad Sci USA. 2011;108(23):9548-9553.

59. Hultqvist M, Olofsson P, Holmberg J, Bäckström BT, Tordsson J, Holmdahl R. Enhanced autoimmunity, arthritis, and encephalomyelitis in mice with a reduced oxidative burst due to a mutation in the Ncf1 gene. Proc Natl Acad Sci USA. 2004;101(34):12646-12651.

60. Frasch SC, et al. NADPH oxidase-dependent generation of lysophosphatidylserine enhances clearance of activated and dying neutrophils via G2A. J Biol Chem. 2008;283(48):33736-33749.

61. Schauer C, et al. Aggregated neutrophil extracellular traps limit inflammation by degrading cytokines and chemokines. Nat Med. 2014;20(5):511-517.

62. Fernandez-Boyanapalli R, et al. Impaired phagocytosis of apoptotic cells by macrophages in chronic granulomatous disease is reversed by IFN- $\gamma$ in a nitric oxide-dependent manner. J Immunol. 2010;185(7):4030-4041.

63. Jackson SH, Devadas S, Kwon J, Pinto LA, Williams MS. T cells express a phagocyte-type NADPH oxidase that is activated after T cell receptor stimulation. Nat Immunol. 2004;5(8):818-827.

64. Richards SM, Clark EA. BCR-induced superoxide negatively regulates B-cell proliferation and T-cell-independent type $2 \mathrm{Ab}$ responses. Eur J Immunol. 2009;39(12):3395-3403.

65. Martinez J, et al. Molecular characterization of LC3-associated phagocytosis reveals distinct roles for Rubicon, NOX2 and autophagy proteins. Nat Cell Biol. 2015;17(7):893-906.

66. Martinez J, et al. Noncanonical autophagy inhibits the autoinflammatory, lupus-like response to dying cells. Nature. 2016;533(7601):115-119.

67. Brinkmann V, Zychlinsky A. Beneficial suicide: why neutrophils die to make NETs. Nat Rev Microbiol. 2007;5(8):577-582.

68. Farrera C, Fadeel B. Macrophage clearance of neutrophil extracellular traps is a silent process. J Immunol. 2013;191(5):2647-2656.

69. Liu CL, et al. Specific post-translational histone modifications of neutrophil extracellular traps as immunogens and potential targets of lupus autoantibodies. Arthritis Res Ther. 2012;14(1):R25.

70. Konig MF, Andrade F. A critical reappraisal of neutrophil extracellular traps and NETosis mimics based on differential requirements for protein citrullination. Front Immunol. 2016;7:461. 